\title{
Aberrant ADAM10 expression correlates with osteosarcoma progression
}

\author{
Ren Zhao ${ }^{1}$, Dongjing $\mathrm{Ni}^{2}$, Yi Tiann $^{2}$, Bing $\mathrm{Ni}^{2^{*}}$ and Aimin Wang ${ }^{1 *}$
}

\begin{abstract}
Background: Osteosarcoma is the most common type of bone cancer and is notorious for its rapid progression. The Notch signaling pathway has recently been shown to be involved in osteosarcoma. As a major sheddase of Notch receptors, ADAM10 has been implicated in many types of cancers, but its role in osteosarcoma has not been investigated. Previous studies have shown that the expression of CD31 was significantly elevated in metastatic osteosarcoma; however, its expression in nonmetastatic groups is not known. In addition, the mysterious multinucleated giant cell in giant cell-rich osteosarcoma was previously regarded as an osteoclast-like cell, but its exact identity is unclear.
\end{abstract}

Method: Tissue chip samples from 40 cases of nonmetastatic osteosarcoma were stained for cytoplasmic ADAM10, activated Notch1 and CD31. Osteoclasts in tumor sections were also stained for tartrate-resistant acid phosphatase (TRAP).

Results: Immunofluorescence staining revealed that ADAM10 expression significantly increased with the progression of osteosarcoma as well as in osteoblastic osteosarcoma, whereas the expression of the Notch intracellular domain (NICD) and CD31 was not significantly altered between different pathological stages. In addition, multinucleated giant cells in giant cell-rich osteosarcoma were also found to coexpress CD31, ADAM10 and NICD, but were negative for TRAP staining.

Conclusions: Our results highlight the importance of ADAM10 in the progression of osteosarcoma and suggest that the protein might be a potential therapeutic target in osteosarcoma treatment. This study also demonstrates that the multinucleated giant cell is an angiogenic tumor cell, rather than an osteoclast, and involves ADAM10/ Notch1 signaling activation.

Keywords: ADAM10, CD31, Multinucleated giant cell, Notch1, Osteoclast, Osteosarcoma, Tumor progression

\section{Background}

Osteosarcoma, the most common type of primary bone cancer, is a malignant neoplasm notorious for its high aggressiveness and early metastatic potential [1]. However, little is known about the signaling pathways that are crucial for its progression, and the molecular biology of osteosarcoma remains poorly understood. Histologically, osteosarcoma is categorized into several different types, including osteoblastic osteosarcoma, chondroblastic osteosarcoma, fibroblastic osteosarcoma and giant

\footnotetext{
* Correspondence: nibingxi@yahoo.com; trauma2@163.com ${ }^{2}$ Institute of Immunology of PLA, Third Military Medical University, No. 30 Gaotanyan Street, Shapingba District, Chongqing 400038, China 'Department of Orthopedics, Daping Hospital, Third Military Medical
University, No. 10 Changjiang Road, Daping, Yuzhong District, Chongqing Department of Orthopedics, Daping Hospital, Third Military Medical
University, No. 10 Changjiang Road, Daping, Yuzhong District, Chongqing 400042, China
}

(c) 2014 Zhao et al.; licensee BioMed Central Ltd. This is an Open Access article distributed under the terms of the Creative Commons Attribution License (http://creativecommons.org/licenses/by/2.0), which permits unrestricted use, distribution, and reproduction in any medium, provided the original work is properly credited. The Creative Commons Public Domain Dedication waiver (http://creativecommons.org/publicdomain/zero/1.0/) applies to the data made available in this article, unless otherwise stated. cell-rich osteosarcoma. The giant cell-rich osteosarcoma is the rare subtype of primary osteogenic sarcoma, and, to the best of our knowledge, only 18 cases have been reported in the literature to date [2]. Its name derives from the existence of multinucleated giant cells within the tumor tissues, which were generally believed to be osteoclast-like tumor cells [2]; yet their exact identity still remains to be elucidated.

A class of disintegrins and metallproteinases, known as ADAMs, have been shown to participate in a variety of signaling events that are aberrant in cancers as well as during tumor progression [3]. ADAM10, a member of the ADAM family, has been found to be upregulated in many cancers, including ovarian, colon and prostate cancers $[4,5]$. Overexpression of ADAM10 promotes the 
growth of oral squamous cell carcinoma and gastric carcinomas, whereas downregulation of its expression reduces proliferation of carcinoma cells [6,7]. However, the role of ADAM10 in osteosarcoma is still unclear.

As a membrane-bound sheddase, ADAM10 cleaves a variety of cell surface proteins, including Notch receptors $[8,9]$. In the canonical Notch signaling pathway, Notch receptors interact with membrane-anchored ligands, followed by sequential proteolytic cleavage by ADAM10 and presenilin within the transmembrane, thus releasing the Notch intracellular domain (NICD), which enters the nucleus and regulates a cohort of Notch-dependent genes. Although aberrant Notch receptor expression has been reported in osteosarcoma [10] and manipulation of the Notch pathway has been shown to play a crucial role for Hes1 (a Notchdependent gene) in osteosarcoma invasion and metastasis $[11,12]$, whether the ADAM10-Notch1 signaling axis is particularly involved in osteosarcoma is unknown.

CD31 is a member of the immunoglobulin (Ig) gene superfamily and plays an important role in a number of endothelial cell functions, including angiogenesis, inflammation, integrin activation and intercellular adhesion [13-15], which could also be employed by tumor cells for their progression. Indeed, on the basis of their immunohistochemical staining of primary and metastatic osteosarcoma samples, Arihiro et al. suggested that the formation of metastatic foci of osteosarcoma cells in other bones might be regulated by CD31 to promote endothelial cell migration [16]. Nevertheless, the role of CD31 in nonmetastatic osteosarcoma has not yet been revealed.

The goal of our present study was to investigate the expression of ADAM10, Notch1, CD31 and tartrateresistant acid phosphatase (TRAP) in nonmetastatic osteosarcoma tissue and their respective contributions to tumor progression.

\section{Methods \\ Tissue chips}

Paraformaldehyde-fixed, paraffin-embedded human osteosarcoma tissue chip slides were purchased from US Biomax (Rockville, MD, USA). Each slide contained 40 duplicate samples of osteosarcoma tissues, and pathological stages included IA $(n=3)$, IB $(n=5)$, IIA $(n=10)$ and IIB $(n=22)$, according to the Musculoskeletal Tumor Society Staging System. This study was approved by the ethics committee of Daping Hospital of the Third Military Medical University.

\section{Reagents and antibodies}

Mouse anti-human CD31 monoclonal antibody (Ab), rabbit anti-human ADAM10 (cytoplasmic domain) polyclonal $\mathrm{Ab}$ and rabbit anti-human activated Notch1 NICD polyclonal $\mathrm{Ab}$ were all purchased from Abcam (Cambridge, UK). Isotype-specific control Abs were also obtained from
Abcam and were used to control staining specificity. Fluorescein isothiocyanate (FITC)-conjugated goat antimouse IgG Ab (ZSGB-BIO, Beijing, China) and cyanine 3 (Cy3)-conjugated goat anti-rabbit IgG Ab (Beyotime Institute of Biotechnology, Jiangsu, China) were used as secondary Abs. Antifade mounting medium was also obtained from Beyotime Institute of Biotechnology.

\section{Immunohistological and immunofluorescent staining}

Paraffin tissue sections were deparaffinized in xylene, rehydrated through graded ethanol series and washed in $10 \mathrm{mM}$ phosphate-buffered saline (PBS), $\mathrm{pH}$ 7.4. Hematoxylin and eosin (H\&E) staining was performed according to a standard protocol. The histological types were assessed by an experienced pathologist specializing in osteosarcoma diagnosis. For immunofluorescent staining, antigen retrieval was performed by incubating tissue sections for 20 minutes in $0.01 \mathrm{M}$ sodium citrate buffer, $\mathrm{pH}$ 6.0, and heated in a microwave oven, then sections were permeabilized with $0.1 \%$ Triton X-100. After blocking with $5 \%$ bovine serum albumin/PBS for 30 minutes, three sequential slides were then incubated with anti-CD31 Ab, anti-ADAM10 (cytoplasmic domain) $\mathrm{Ab}$ and anti activated Notch1 (NICD) Ab, respectively. For double-immunofluorescence staining, two additional sequential slides were incubated with either anti-CD31 Ab (mouse anti-human) plus anti-ADAM10 Ab (rabbit anti-human) or anti-CD31 Ab plus anti-NICD Ab (rabbit anti-human), respectively. Isotype-specific control Abs were used to test staining specificity. Slides were incubated overnight at $4^{\circ} \mathrm{C}$. After being washed with PBS, slides were stained with secondary FITC anti-mouse Ab and Cy3 anti-rabbit $\mathrm{Ab}$ for 30 minutes at room temperature (RT). Afterward, nuclei were stained with 4',6-diamidino-2-phenylindole (DAPI) for 10 minutes at RT. Images were obtained using the MRC-600 digital confocal laser scanning system (Bio-Rad Laboratories, Hercules, CA, USA).

\section{TRAP/DAPI staining}

TRAP staining was performed on deparaffinized and rehydrated sections using the Acid Phosphatase, Leukocyte (TRAP) Kit (Sigma-Aldrich, St Louis, MO, USA) according to the manufacturer's instructions. DAPI staining was performed afterward as mentioned above to visualize the nuclei.

\section{Data analysis}

Acquired images were exported and merged using ImagePro Plus version 6.0 software (MediaCybernetics, Rockville, MD, USA). NICD and CD31 density percentages were measured as the percentage of positive FITC signal area against the whole field area under $100 \times$ magnification as described by Connor et al. [17]. ADAM10-positive tumor cells were counted in ADAM10/DAPI merged images 
under the same magnification, and analysis was performed with tools in Image-Pro Plus software. Statistical differences were determined using a two-tailed, unpaired Student's $t$-test.

\section{Results}

ADAM10 expression was significantly increased in more advanced and osteoblastic osteosarcoma

We first examined the expression of ADAM10 in osteosarcoma tissue chips. Under $400 \times$ magnification, a polarized and condensed expression pattern of cytoplasmic
ADAM10 was observed in all cases and stages of osteosarcoma (Figure 1). In order to determine whether ADAM10 was differentially expressed among different stages of osteosarcoma, $\mathrm{ADM} 10^{+}$tumor cells were counted under 100× ADAM10/DAPI merged images. The number of $\mathrm{ADM}^{+} 0^{+}$tumor cells in stages IIA and IIB increased significantly compared with those in stage IA (Figure 1), suggesting that elevated ADAM10 levels in tumor cells might be involved in the progression of osteosarcoma.

After the immunofluorescent staining, osteosarcoma tissue chips were stained with H\&E. Four types of

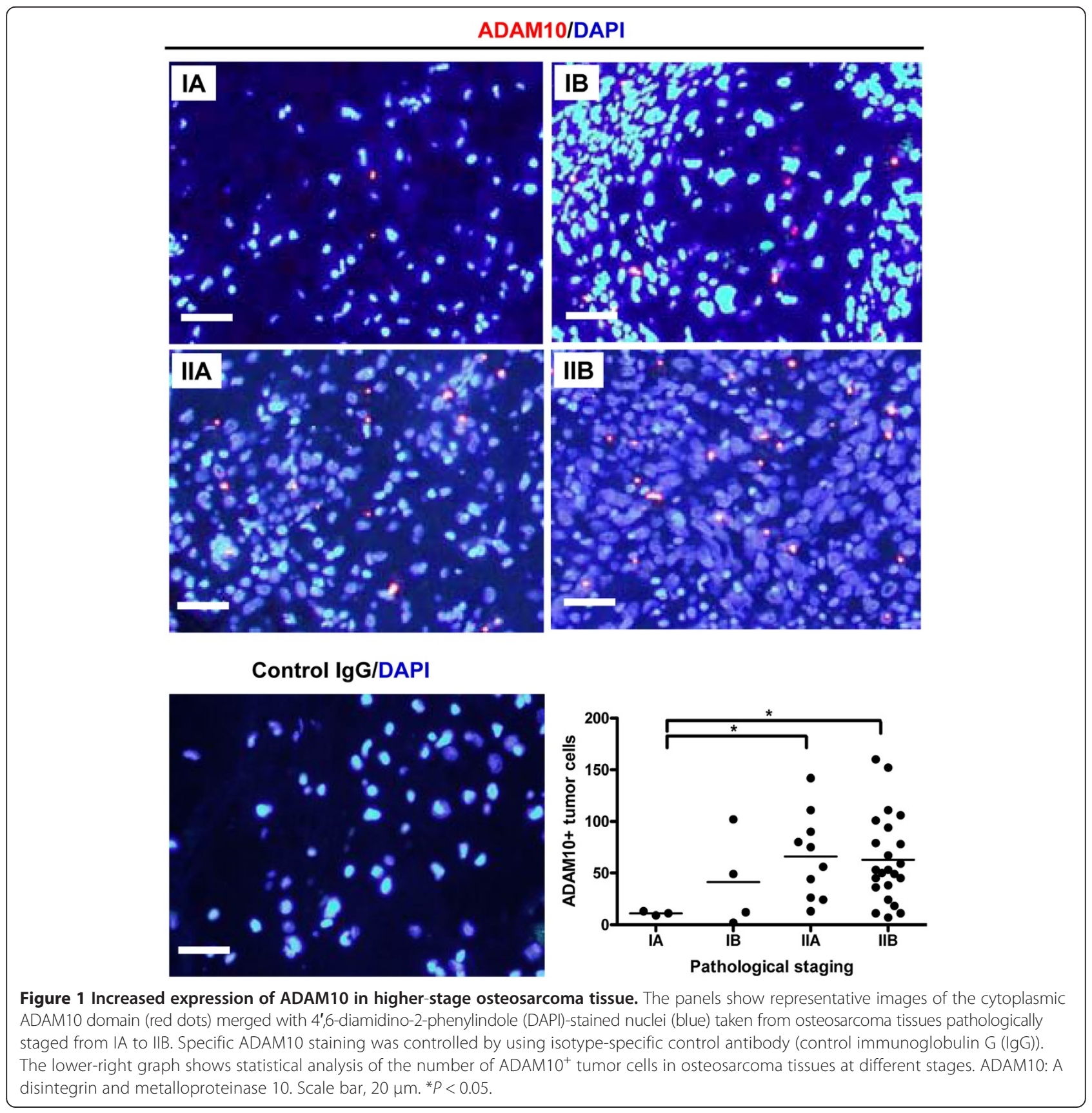


osteosarcoma were recognized, including giant cellrich osteosarcoma, osteoblastic osteosarcoma, fibroblastic osteosarcoma and chondroblastic osteosarcoma (Additional file 1: Figure S1, upper panels). We then reanalyzed the number of ADAM $10^{+}$tumor cells based on different histological types and found that osteoblastic osteosarcoma had significantly more ADAM10 ${ }^{+}$ tumor cells compared with chondroblastic and fibroblastic osteosarcomas (Additional file 1: Figure S1, lower graph), suggesting that ADAM10 might be involved specifically in the pathogenesis of osteoblastic osteosarcoma.
Activated Notch1 was not markedly altered in different stages of osteosarcoma

As ADAM10 is a major regulator of Notch signaling via its shedding of Notch receptors [8,9], we sought to determine whether increased expression of ADAM10 in osteosarcoma progression would also lead to increased activation of Notch1. We detected the expression of activated Notch1 (NICD) in another sequential slide. As shown in the upper panels of Figure 2, Notch1 was activated in osteosarcoma tissue at all stages; however, its activation was not significantly correlated with pathological staging, as shown by NICD density from

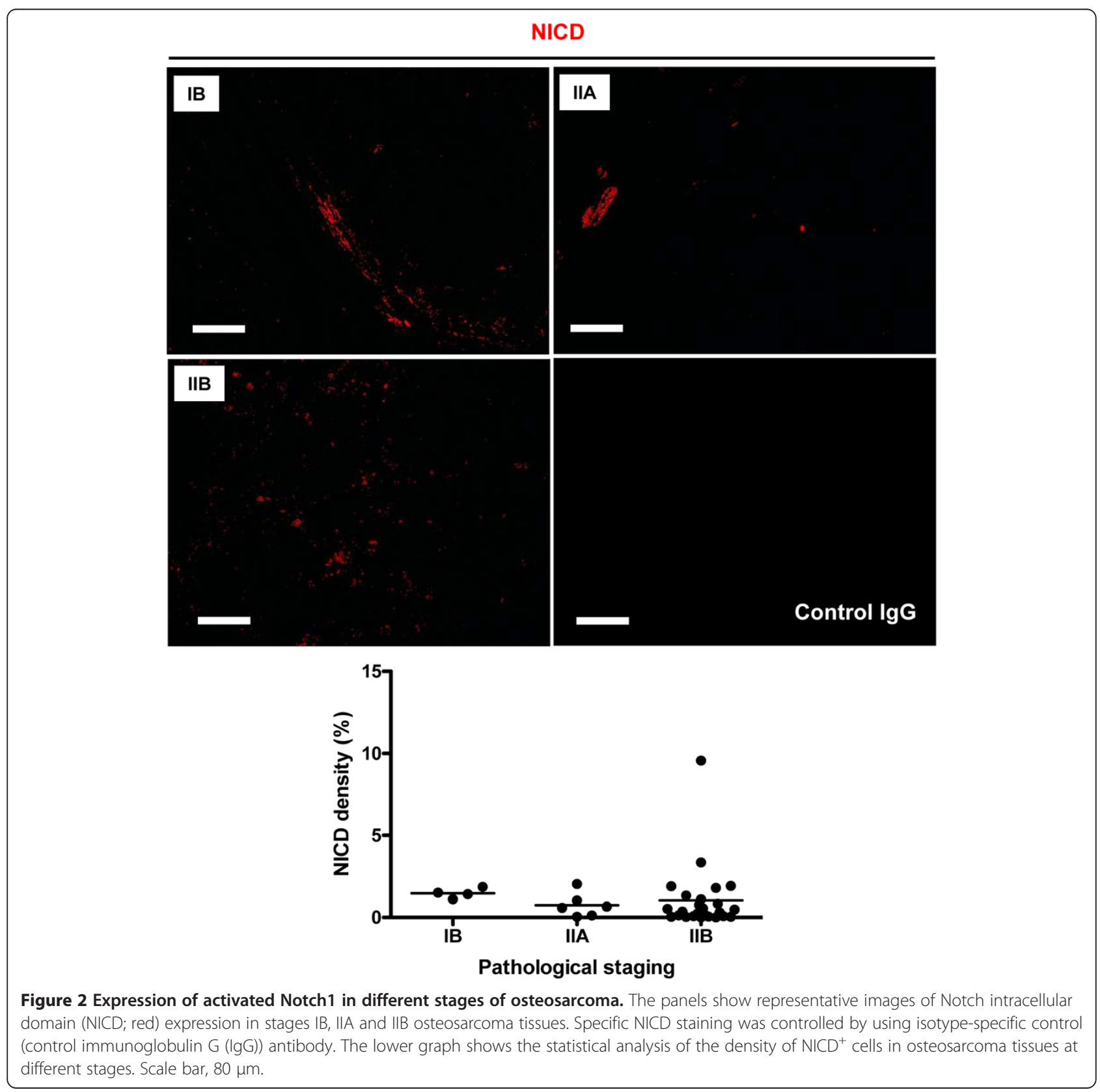


stage IB to stage IIB. These results suggest that Notch1 was activated in osteosarcoma but that its activation might not contribute to disease progression.

\section{CD31 might not contribute to osteosarcoma progression} Previous studies have shown that the expression of CD31 was significantly increased in metastatic osteosarcoma compared with primary osteosarcoma [16]. However, the differences between nonmetastatic groups are unclear. Hence we performed FITC-CD31 staining on all osteosarcoma tissues from stages IA to IIB. Although a trend of increased CD31 expression was observed as osteosarcoma progressed, no significant differences were found between different pathological stages in terms of CD31 density (Figure 3), suggesting that vascularity (represented by the expression of CD31) was not dramatically altered during the pathological progression of nonmetastatic osteosarcoma, a scenario different from metastatic groups.

\section{Multinucleated giant cell is angiogenic tumor cell, not osteoclast}

We found that the multinucleated giant cells in giant cellrich osteosarcoma stained positively with CD31 (Figure 4, left panels), suggesting their angiogenic property. Moreover, cytoplasmic ADAM10 and activated Notch1 were colocalized within these giant cells (Figure 4), suggesting

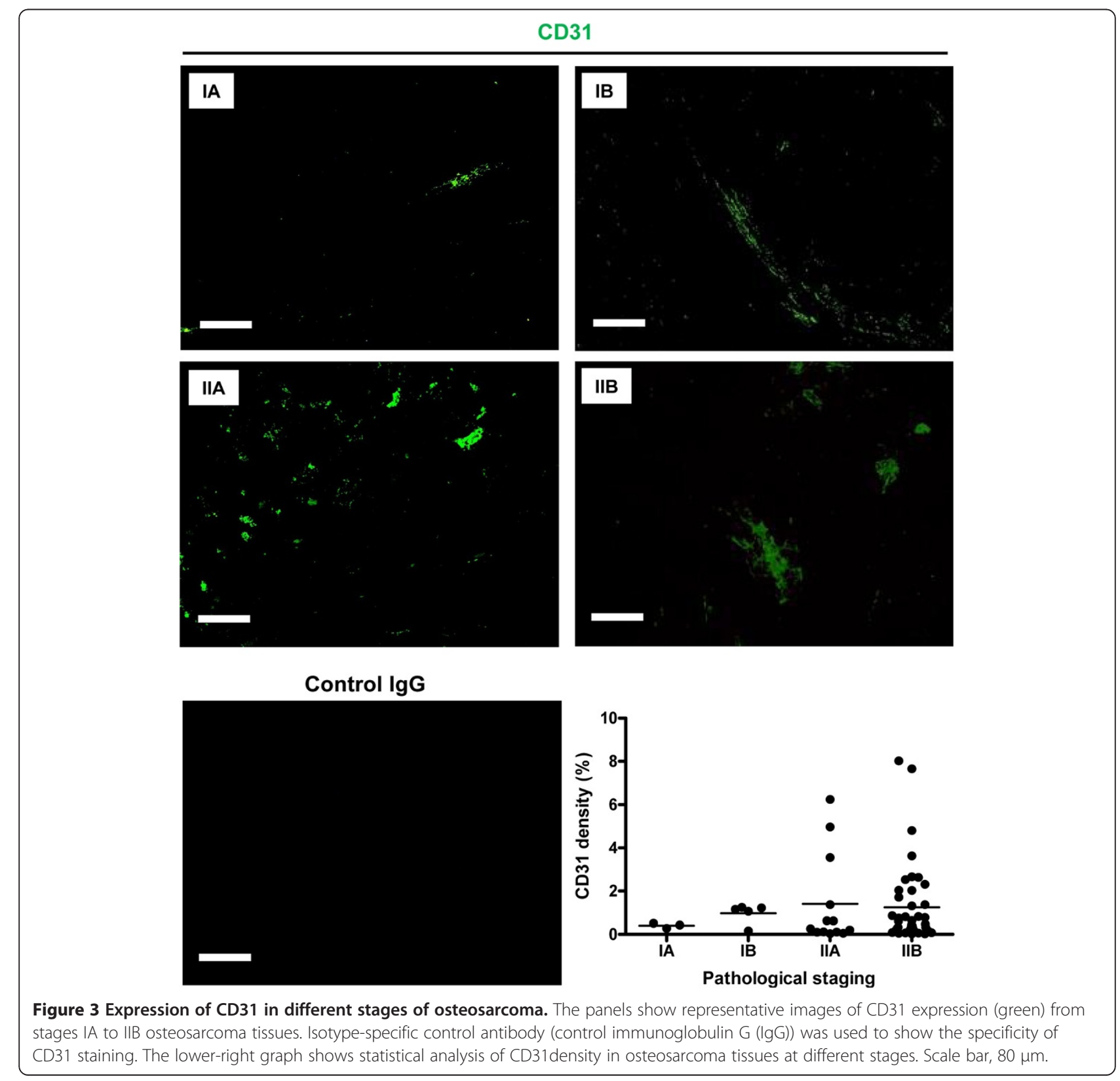




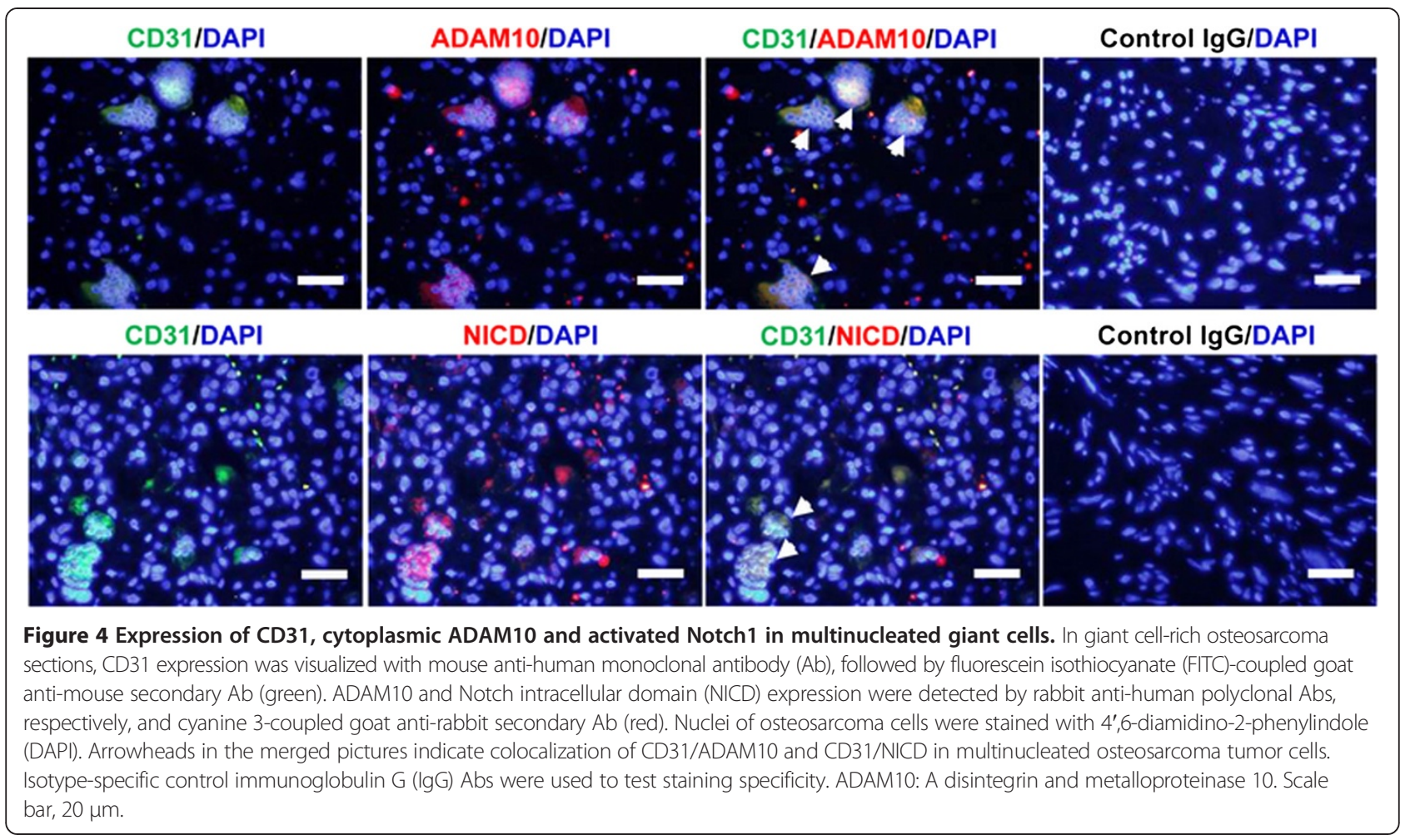

that ADAM10/Notch1 signaling was activated in these angiogenic tumor cells. By TRAP staining, we observed that TRAP was absent in multinucleated cells in giant cell-rich osteosarcoma tissue, whereas $\mathrm{TRAP}^{+}$cells were found in osteoblastic, fibroblastic and chondroblastic osteosarcoma tissue (Figure 5). Taken together, these results suggest that what were previously called osteoclast-like multinucleated giant cells [2] are not osteoclasts, but rather angiogenic tumor cells that involve ADAM10/Notch1 signaling activation.

\section{Discussion}

Although ADAM10 has been found to be upregulated in many cancers $[4,5]$, its expression in tumors with mesenchymal origin (particularly osteosarcoma) remains unknown. Herein we show that ADAM10 was generally expressed in osteosarcoma tumor cells in a condensed and polarized pattern. The level of ADAM10 in tumor cells significantly increases as osteosarcoma progresses, as well as in osteoblastic osteosarcoma tissue, suggesting that it might play a role in osteosarcoma tumor progression and the pathological development of osteoblastic osteosarcoma. As ADAM10 is a major sheddase of Notch receptors $[8,9]$, and because researchers in recent studies have discovered that Notch signaling was activated in human osteosarcoma and may play a role in tumor invasion and metastasis $[11,18,19]$, we speculate that ADAM10 might participate in osteosarcoma progression through the shedding of Notch receptors and activation of the Notch signaling pathway.

Currently, there is no systematic evaluation comparing Notch expression in clinical osteosarcoma between different pathological stages. Hence, we analyzed activated Notch1 (NICD) expression from stages IB to IIB at the protein level and found no significant alteration in NICD density. Therefore, as one of four members in the mammalian Notch receptor family, Notch1 might not be the Notch receptor responsible for osteosarcoma progression. Similarly, Tanaka et al. found that expression of Notch1 mRNA was decreased in specimens from human osteosarcoma biopsies compared with normal bone tissues [20].

Previous studies have shown that CD31 expression was significantly correlated between primary and metastatic osteosarcomas [16], but the correlation between nonmetastatic groups is not clear. In our present study, we found that variation in the density of CD31 was not significantly associated with osteosarcoma staging from IA to IIB, although there was a trend toward increased CD31 expression as osteosarcoma progressed.

The expression of CD31 and activation of Notch1 did not dramatically contribute to osteosarcoma progression overall. In the specific case of multinucleated tumor cells in giant cell-rich osteosarcoma, however, the expression of CD31 may facilitate the adhesion of these tumor cells to endothelial cells. Migration between endothelial cells may also occur via homophilic interactions between CD31 and 

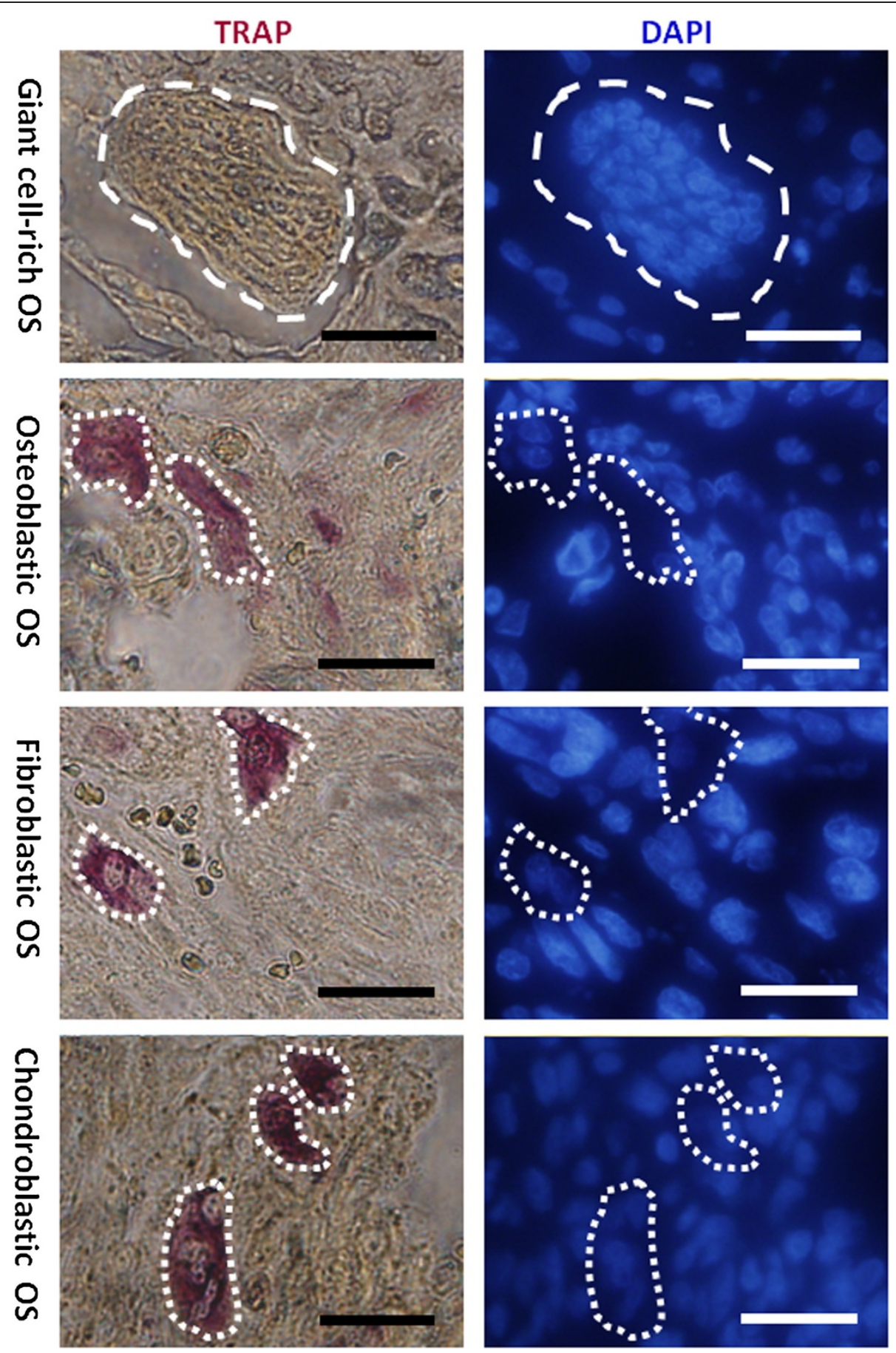

Figure 5 TRAP/DAPI staining of various histological types of osteosarcoma. The left panels show tartrate-resistant acid phosphatase (TRAP) staining of giant cell-rich, osteoblastic, fibroblastic and chondroblastic osteosarcomas, respectively. The right panels show 4',6-diamidino-2-phenylindole (DAPI) staining of the nuclei of sections identical to those on the left panels. Note that negative TRAP staining was observed in multinucleated giant cells (upper panel). Dashed lines outline the cell contours. Scale bar, $20 \mu \mathrm{m}$.

CD31 and heterophilic interactions between CD31 and $\alpha_{v} \beta_{3}$ integrin, which has been shown to be expressed in both osteosarcoma cells and endothelial cells [14,21,22], thus promoting tumor cell migration. Given the fact that these tumor cells also coexpress ADAM10 and activated Notch1, ADAM10/Notch1 signaling might also participate in this process, as recent studies have demonstrated that ADAM10 and Notch1 could promote the migration of a 
variety of tumor cells, both cooperatively [23] and individually $[24,25]$. Furthermore, the absent staining of TRAP and positive staining of CD31 in multinucleated giant cells suggest that these cells are not osteoclasts, but rather a type of angiogenic tumor cells in which ADAM10/Notch1 signaling is activated.

\section{Conclusion}

In our present study, we examined the expression of CD31, ADAM10, Notch1 and TRAP in nonmetastatic osteosarcoma tissue chips. We found that only ADAM10 expression was significantly correlated with tumor progression and osteoblastic osteosarcoma development. Our results also suggest that multinucleated giant cells are not osteoclasts, but angiogenic tumor cells in which ADAM10/ Notch1 signaling is activated and might be implicated in tumor cell migration. The results of this study imply heterogeneity in osteosarcoma between metastatic and nonmetastatic types and complexity within nonmetastatic types. It also highlights ADAM10 as a potential target for the biological intervention of nonmetastatic osteosarcoma.

\section{Additional file}

Additional file 1: Figure S1. Analysis of $\mathrm{ADAM}^{+} 0^{+}$cells in various histological types of osteosarcoma. The upper panels show hematoxylin and eosin staining of giant cell-rich, osteoblastic, fibroblastic, and chondroblastic osteosarcoma tissue, respectively. The lower graph shows the number of tumor cells expressing ADAM10 in chondroblastic, fibroblastic, and osteoblastic osteosarcoma tissue. Scale bar, $20 \mu \mathrm{m}$. NS, not significant ${ }^{*} P<0.05,{ }^{* *} P<0.001$.

\section{Competing interests}

The authors declare that they have no competing interests.

\section{Authors' contributions}

ZR designed the study, carried out the immunohistological and immunofluorescence studies and TRAP staining experiments, collected and analyzed the data and drafted the manuscript. NDJ participated in the immunofluorescence experiment. TY helped to analyze the data. NB and WAM participated in the study design and revised the manuscript. All authors read and approved the final manuscript.

\section{Acknowledgements}

We thank Dr Cheng Bian and Dr Haitao Zhu for their constructive suggestions and Dr Hongkai Wang for his generous gift of the TRAP kit. We also appreciate Dr Sangao Fang and Ying Zeng for their help with histological typing to identify osteosarcoma tissues.

Received: 9 September 2013 Accepted: 7 February 2014 Published: 18 February 2014

\section{References}

1. Messerschmitt PJ, Garcia RM, Abdul-Karim FW, Greenfield EM, Getty PJ: Osteosarcoma. J Am Acad Orthop Surg 2009, 17:515-527.

2. Wang CS, Yin QH, Liao JS, Lou JH, Ding XY, Zhu YB: Giant cell-rich osteosarcoma in long bones: clinical, radiological and pathological features. Radiol Med 2013, 118:1324-1334.

3. Mochizuki S, Okada Y: ADAMs in cancer cell proliferation and progression. Cancer Sci 2007, 98:621-628.

4. Arribas J, Bech-Serra JJ, Santiago-Josefat B: ADAMs, cell migration and cancer. Cancer Metastasis Rev 2006, 25:57-68.
5. Santiago-Josefat B, Esselens C, Bech-Serra JJ, Arribas J: Post-transcriptional up-regulation of ADAM17 upon epidermal growth factor receptor activation and in breast tumors. J Biol Chem 2007, 282:8325-8331.

6. Carl-McGrath S, Lendeckel U, Ebert M, Roessner A, Röcken C: The disintegrin-metalloproteinases ADAM9, ADAM12, and ADAM15 are upregulated in gastric cancer. Int J Oncol 2005, 26:17-24.

7. Ko SY, Lin SC, Wong YK, Liu CJ, Chang KW, Liu TY: Increase of disintegrin metalloprotease 10 (ADAM10) expression in oral squamous cell carcinoma. Cancer Lett 2007, 245:33-43.

8. Bozkulak EC, Weinmaster G: Selective use of ADAM10 and ADAM17 in activation of Notch1 signaling. Mol Cell Biol 2009, 29:5679-5695.

9. van Tetering $G$, van Diest $P$, Verlaan I, van der Wall E, Kopan R, Vooijs M: Metalloprotease ADAM10 is required for Notch1 site 2 cleavage. J Biol Chem 2009, 284:31018-31027.

10. Engin $F$, Bertin $T$, Ma $O$, Jiang MM, Wang L, Sutton RE, Donehower $L A$, Lee $B$ : Notch signaling contributes to the pathogenesis of human osteosarcomas. Hum Mol Genet 2009, 18:1464-1470.

11. Zhang P, Yang Y, Zweidler-McKay PA, Hughes DP: Critical role of notch signaling in osteosarcoma invasion and metastasis. Clin Cancer Res 2008, 14:2962-2969.

12. Hughes DP: How the NOTCH pathway contributes to the ability of osteosarcoma cells to metastasize. Cancer Treat Res 2009, 152:479-496.

13. Muller WA, Weigl SA, Deng X, Phillips DM: PECAM-1 is required for transendothelial migration of leukocytes. J Exp Med 1993, 178:449-460.

14. Buckley CD, Doyonnas R, Newton JP, Blystone SD, Brown EJ, Watt SM, Simmons DL: Identification of $\alpha_{v} \beta_{3}$ as a heterotypic ligand for CD31/ PECAM-1. J Cell Sci 1996, 109:437-445.

15. Dejana E, Zanetti A, Del Maschio A: Adhesive proteins at endothelial cell-to-cell junctions and leukocyte extravasation. Haemostasis 1996, 26(Suppl 4):210-219.

16. Arihiro K, Inai K: Expression of CD31, Met/hepatocyte growth factor receptor and bone morphogenetic protein in bone metastasis of osteosarcoma. Pathol Int 2001, 51:100-106.

17. Connor KM, Krah NM, Dennison RJ, Aderman CM, Chen J, Guerin KI, Sapieha P, Stahl A, Willett KL, Smith LE: Quantification of oxygen-induced retinopathy in the mouse: a model of vessel loss, vessel regrowth and pathological angiogenesis. Nat Protoc 2009, 4:1565-1573.

18. Engin F, Yao Z, Yang T, Zhou G, Bertin T, Jiang MM, Chen Y, Wang L, Zheng $H$, Sutton RE, Boyce BF, Lee B: Dimorphic effects of Notch signaling in bone homeostasis. Nat Med 2008, 14:299-305

19. Zhang P, Yang Y, Nolo R, Zweidler-McKay PA, Hughes DP: Regulation of NOTCH signaling by reciprocal inhibition of HES1 and Deltex1 and its role in osteosarcoma invasiveness. Oncogene 2010, 29:2916-2926. A published erratum appears in Oncogene 2012, 31:4732.

20. Tanaka M, Setoguchi T, Hirotsu M, Gao H, Sasaki H, Matsunoshita Y, Komiya S: Inhibition of Notch pathway prevents osteosarcoma growth by cell cycle regulation. Br J Cancer 2009, 100:1957-1965.

21. Stuiver I, Ruggeri $Z$, Smith JW: Divalent cations regulate the organization of integrins $\alpha_{v} \beta_{3}$ and $a_{v} \beta_{5}$ on the cell surface. J Cell Physiol 1996, 168:521-531.

22. Lanza P, Felding-Habermann B, Ruggeri ZM, Zanetti M, Billetta R: Selective interaction of a conformationally-constrained Arg-Gly-Asp (RGD) motif with the integrin receptor $a_{v} \beta_{3}$ expressed on human tumor cells. Blood Cells Mol Dis 1997, 23:230-241.

23. Guo J, He L, Yuan P, Wang P, Lu Y, Tong F, Wang Y, Yin Y, Tian J, Sun J: ADAM10 overexpression in human non-small cell lung cancer correlates with cell migration and invasion through the activation of the Notch1 signaling pathway. Oncol Rep 2012, 28:1709-1718.

24. Lee SB, Schramme A, Doberstein K, Dummer R, Abdel-Bakky MS, Keller S, Altevogt P, Oh ST, Reichrath J, Oxmann D, Pfeilschifter J, Mihic-Probst D, Gutwein P: ADAM10 is upregulated in melanoma metastasis compared with primary melanoma. J Invest Dermatol 2010, 130:763-773.

25. Bolós V, Mira E, Martínez-Poveda B, Luxán G, Cañamero M, Martínez-A C, Mañes S, de la Pompa JL: Notch activation stimulates migration of breast cancer cells and promotes tumor growth. Breast Cancer Res 2013, 15:R54.

doi:10.1186/2047-783X-19-9

Cite this article as: Zhao et al: Aberrant ADAM10 expression correlates with osteosarcoma progression. European Journal of Medical Research 2014 19:9. 